\title{
Crecimiento y comportamiento reproductivo de la gallina criolla de huevos con cáscara verde de la provincia de Chota, Cajamarca
}

\section{Growth and reproductive performance of the creole hen of green shell eggs of the Province of Chota, Cajamarca}

\author{
Manuel Paredes A. ${ }^{1,2}$, Alcira Romero C. ${ }^{1}$, Magaly Torres R. ${ }^{1}$, \\ Luis Vallejos F. ${ }^{1}$, José Mantilla G. ${ }^{1}$
}

\section{Resumen}

El estudio tuvo por objetivo evaluar el crecimiento y comportamiento reproductivo de la gallina criolla de huevo con cáscara verde procedente de la provincia de Chota, Cajamarca (Perú). Se colectaron 500 huevos y se incubaron artificialmente para determinar su incubabilidad. En los pollos resultantes se evaluó el crecimiento en confinamiento durante 16 semanas. Luego, las aves fueron apareadas (5 hembras y 1 macho) y se determinó la producción y peso del huevo de seis biotipos e incubabilidad bajo incubación natural. Se encontraron diferencias significativas $(p<0.05)$ en el crecimiento y tamaño de los seis biotipos evaluados. La gallina inició la ovoposición entre 17 y 19 semanas de edad, con periodos de postura de siete semanas interrumpidos por una etapa de cloquera que puede durar hasta tres semanas. La incubabilidad se vio influenciada por las condiciones y tipos de incubación.

Palabras clave: gallina criolla; huevo de cáscara verde; crecimiento; reproducción

\section{AbSTRaCT}

The aim of this study was to evaluate the productive and reproductive performance of the Creole egg hen of green shell eggs of the province of Chota, Cajamarca (Peru). Five hundred eggs were collected and artificially incubated to determine their hatchability. In the resulting chicks, the growth in confinement was evaluated for 16 weeks. Then, the

\footnotetext{
${ }^{1}$ Departamento Académico de Ciencias Pecuarias, Facultad de Ingeniería en Ciencias Pecuarias, Universidad Nacional de Cajamarca, Perú

${ }^{2}$ E-mail: mepaunc@gmail.com mparedes@unc.edu.pe
}

Recibido: 11 de julio de 2018

Aceptado para publicación: 12 de febrero de 2019 
birds were mated ( 5 females and 1 male) and the production and weight of eggs of six biotypes and the hatchability were determined under natural incubation. Significant differences $(p<0.05)$ were found in the growth and size of the six biotypes evaluated. The birds initiated the oviposition between 17 and 19 weeks of age, with periods of posture of seven weeks interrupted by a broody period that could last up to three weeks. The hatchability was influenced by the conditions and types of incubation.

Key words: creole hen; green shell egg; growth; reproduction

\section{INTRODUCCIÓN}

La demanda de alimentos nativos y saludables por parte de un sector creciente de consumidores permite que la agricultura orgánica alcance mayor desarrollo en los últimos años. Dentro del promisorio sector de alimentos orgánicos, el huevo de aves de corral, enriquecido con ácidos grasos omega 3 es un alimento muy ofertado y de importancia en la nutrición humana (Farrel, 2008), sobre todo en personas con tendencia a padecimientos cardiovasculares.

En gallinas de líneas comerciales bajo sistemas productivos al pastoreo se ha logrado incrementar los depósitos de carotenoides en la yema y de ácidos grasos poliinsaturados (PUFA) n-3 con relación al porcentaje de PUFA n-6 (Mugnai et al., 2014). También se ha mejorado el contenido de PUFA en el huevo con la adición de alimentos ricos en omega-3 como linaza, aceite de pescado y otros ingredientes (Dunn-Horrocks et al., 2011). Es así que la interacción sistema de producción y contenido nutricional del huevo tiene el potencial de modificar la naturaleza, propiedades biológicas, químicas y físicas de este alimento (Wilson, 2017).

Los fundamentos de la mercadotecnia establecen que la presentación del producto, sobre todo el empaque, influye positivamente en la adquisición y aceptación de un producto; en tal sentido el empaque natural y visible del huevo es su cáscara. Se ha determinado que el tiempo de oviposición afecta la intensidad del color de la cáscara, siendo más oscuros los huevos puestos en las primeras horas del día y más claros en horas avanzadas de la mañana. La intensidad del color disminuye, además, con la edad de la gallina (Samiullah et al., 2016). También se han cuantificado las concentraciones de los pigmentos de la cáscara del huevo, siendo la biliverdina el pigmento que predomina sobre la protoporfirina en cáscaras de huevo color verde, sucediendo lo contrario con el huevo de cáscara marrón (Wang et al., 2009).

La gallina criolla ha sido estudiada como reservorio genético natural a fin de salvaguardar la variabilidad genética de la especie, habiéndose encontrado hasta 10 biotipos de gallinas en comunidades ecuatorianas (Villacís et al., 2014). Sin embargo, aún existen numerosos detalles que se desconocen de las gallinas domésticas (Gallus gallus) sobre su domesticación, origen, evolución y conservación de germoplasma (Osman et al., 2016). Por tanto, se requiere profundizar el conocimiento del comportamiento productivo y reproductivo de las gallinas criollas de procedencias locales productoras de huevo con cáscara verde, incluyendo la relación entre el valor nutritivo del huevo con su color de cáscara (Samiullah et al., 2016).

El presente estudio tuvo por objetivo evaluar indicadores productivos de crecimiento y comportamiento reproductivo de la gallina criolla productora de huevos con cáscara verde, procedente de la crianza traspatio que se da en pequeños poblados del Perú, ubicados en la provincia andina de Chota, de la región Cajamarca. 


\section{Materiales y Métodos}

\section{Huevos}

Se recolectaron 500 huevos de gallina con cáscara verde en sus diferentes tonalidades, procedentes de 72 familias rurales de los centros poblados Chuyabamba, Marcopampa, Pampa Grande, Campamento y Churucancha, ubicados en un radio de 10 km alrededor de la capital del distrito de Chota (2388 msnm), provincia de Chota, región Cajamarca (Perú). La fase de recolección de huevos se hizo durante siete días. Los huevos fueron pesados en balanza electrónica colgante PCE-CS de $1000 \mathrm{~g}$ de capacidad y $0.01 \mathrm{~g}$ de precisión, y almacenados en bandejas de cartón de 30 x $30 \mathrm{~cm}$.

La incubación de los huevos se realizó en la provincia costera de Trujillo, en una empresa comercial, ubicada a $400 \mathrm{~km}$ de Chota, tras un recorrido de 9 horas por carretera. Los huevos fueron pesados y colocados en una incubadora de etapa única (Jamesway, Canadá), durante 18 días, a temperatura de $37.5^{\circ} \mathrm{C}$ y humedad relativa de $85 \%$. Los huevos fueron transferidos a una nacedora Jamesway a los 18 días de incubación, y colocados en bandejas de incubación hasta los 21.5 días, distribuidos en 18 huevos por sección.

Se determinó el porcentaje de incubabilidad de acuerdo con el número de pollos nacidos con relación a los 500 huevos incubados. Los huevos no eclosionados (HNE) fueron abiertos para determinar las causas de la no eclosión, registrándose el porcentaje de huevos putrefactos en estado acuoso (HPEA) con relación al total de HNE. También se determinó el porcentaje de mortalidad embrionaria al final de la incubación con relación al total de huevos incubados. Los 390 polluelos resultantes fueron empacados en cuatro cajas convencionales de cartón y llevados vía terrestre a la granja experimen- tal de la Facultad de Ingeniería en Ciencias Pecuarias de la Universidad Nacional de Cajamarca, ubicada en Cajamarca a 2750 msnm.

\section{Aves de 0-10 Semanas}

Las aves fueron asignadas aleatoriamente a 10 corrales, divididos por cercas circulares de cría, con viruta como material de cama sobre piso de concreto, en un galpón de lados abiertos con cortinas laterales. Cada corral representó una repetición (39 aves por repetición). El espacio durante la primera semana fue de $0.038 \mathrm{~m}^{2}$ por ave. No se hizo el sexado inicial de los pollos. Cada corral estaba equipado con un comedero tipo bandeja y un bebedero de un galón de capacidad y tuvieron $24 \mathrm{~h}$ de iluminación. Se proporcionó alimento en forma de harina y agua a voluntad. La temperatura del galpón se mantuvo a $32{ }^{\circ} \mathrm{C}$ durante la primera semana, reduciéndose $3{ }^{\circ} \mathrm{C}$ por semana hasta alcanzar $20^{\circ} \mathrm{C}$ a las cinco semanas, donde se retiraron los equipos de calefacción, quedando expuestas las aves a temperatura ambiente. Los comederos y bebederos para pollos BB fueron cambiados por dos comederos tipo tolva y un bebedero tipo campana, ambos de plástico y colgantes, por cada corral, a los 10 días de edad.

Las dietas de inicio, crecimiento y desarrollo se dieron entre 1 y 42,43 y 84, y 85 y 112 días de edad, respectivamente. Las dietas cumplieron con los estándares de requerimientos de nutrientes para pollas en crecimiento de líneas comerciales de postura, sugeridas por NRC (1994) (Cuadro 1). Las aves fueron vacunadas con Poulvac Ovoline CVI + HVT, Newcastle B1B1 + Bronquitis Mass y Poulvac TRT el día 1 en la planta de incubación, con Bursine-2 y Newcastle La Sota + Bronquitis Mass el día 14 y con Newcastle La Sota + Bronquitis Mass en la semana 6. Todas las vacunas procedieron de la empresa Montana (Perú). 


\section{Aves de 10-16 Semanas}

Las aves fueron sexadas a las $10 \mathrm{se}-$ manas por características morfológicas (los machos tienen más desarrollada la cresta y de color rojo más intenso) (Quintana, 2013). Se desecharon pollos con defectos físicos, aspecto débil y de bajo peso corporal. Las aves $(n=300)$ fueron reagrupadas y asignadas aleatoriamente a 10 corrales por sexo (150 hembras, 150 machos), divididos por cercas rectangulares de malla de alambre galvanizado, con viruta como material de cama sobre piso de concreto, representando cada corral a una repetición ( 15 aves por repetición). El espacio fue de 3 aves $/ \mathrm{m}^{2}$. Se controló el peso de las aves con una balanza KERN EW 60001M (Alemania) de $6000 \mathrm{~g}$ de capacidad y $0.1 \mathrm{~g}$ de precisión de lectura. Se determinó la ganancia media diaria (GMD) y el consumo de alimento (se registró el suministro y residuo diario), así como la conversión alimenticia por repetición en la primera etapa (1-10 semanas) y de acuerdo con el sexo (11-16 semanas) considerando la ganancia de peso y el consumo de alimento. La mortalidad fue expresada en porcentaje del total de aves al inicio de la evaluación (390 pollos BB).

\section{Aves de 16-33 Semanas}

A las 16 semanas de edad se determinaron los genotipos presentes con base al color de plumaje, presencia de plumaje en el cuello y tamaño de las aves, encontrándose seis biotipos, tanto en machos como en hembras: negro, castaño, colorado, blanco, cuello desnudo y tipo riña. Las aves fueron trasladadas a 24 corrales de $3 \mathrm{~m}^{2}$ cada uno (cinco gallinas y un gallo por corral), siendo cuatro corrales por genotipo, definiéndose un experimento con seis tratamientos (biotipos) y cada tratamiento con cuatro repeticiones (corrales), con 120 gallinas y 24 gallos. Las gallinas fueron alojadas experimentalmente de 17 a 33 semanas de edad (marzo-mayo, 2013). Cada ave tenía acceso a espacios de $0.50 \mathrm{~m}^{2}$. Los corrales estuvieron divididos por redes y los espacios interiores estaban pro- vistos de nidos de madera y perchas. El piso estaba cubierto con viruta de madera.

La alimentación de las aves en la semana 17 fue con un pienso concentrado de prepostura $(\mathrm{PC}=17.0 \% ; \mathrm{EM}=2900 \mathrm{kcal} / \mathrm{kg})$. En la semana 18 (inicio de la postura en algunas aves), se cambió al pienso de postura (Cuadro 1), el cual se mantuvo durante el resto del periodo experimental (semanas 18-33). El alimento fue proporcionado ad libitum.

La temperatura ambiental y humedad relativa fue medida durante todo el periodo experimental con un higrómetro térmico TIGITH, LSI. Las condiciones termohigrométricas interiores y exteriores siguieron la misma tendencia. La temperatura media fue de $19 \pm 5^{\circ} \mathrm{C}$ y $15 \pm 7^{\circ} \mathrm{C}$, y la humedad relativa media fue de $65 \pm 3 \%$. La ovoposición se produjo en horas de la noche y primeras horas del día, mayoritariamente. $\mathrm{El}$ fotoperiodo fue natural. La intensidad de la luz varió de 20 a 90 lux de acuerdo con la hora del día y las condiciones climáticas

\section{Postura e Incubabilidad de Huevos}

Los huevos fueron retirados a las 08:00 y 10:00 cada día y fueron pesados (balanza de precisión de $6000 \mathrm{~g}$ de capacidad y $0.1 \mathrm{~g}$ de precisión KERN EW 6000). Los huevos producidos durante la primera fase de postura, una vez contados y pesados, fueron desechados, no considerándose para la incubación. El primer periodo de postura se vio interrumpido por un periodo de cloquera, donde se suministró alimento de crecimiento durante tres semanas, luego de lo cual se restituyó el alimento de postura. El número de huevos producidos durante el periodo de cloquera no superó el $10 \%$ de la producción previa en ningún biotipo.

Los huevos producidos durante las dos últimas semanas de postura de la segunda fase se colocaron en bandejas de cartón y se almacenaron en un ambiente con paredes y techo de cemento a temperatura ambiental $\left(8 \pm 3{ }^{\circ} \mathrm{C}\right)$. Al término de la postura e iniciada la segunda fase de cloquera se seleccio- 
Cuadro 1. Ingredientes y análisis calculado de las dietas (base fresca) proporcionadas a aves criollas chotanas de huevos verdes

\begin{tabular}{|c|c|c|c|c|c|}
\hline Ingredientes (g/kg) & $\begin{array}{l}\text { Iniciador } \\
(0-6 \text { sem })\end{array}$ & $\begin{array}{l}\text { Crecimiento } \\
(7-12 \mathrm{sem})\end{array}$ & $\begin{array}{c}\text { Desarrollo } \\
\text { (12-16 } \\
\text { sem) }\end{array}$ & $\begin{array}{c}\text { Pre- } \\
\text { postura } \\
(17 \mathrm{sem})\end{array}$ & $\begin{array}{c}\text { Postura } \\
(17-33 \\
\text { sem })\end{array}$ \\
\hline Maíz amarillo & 527 & 484 & 498 & 489 & 570 \\
\hline Harina de pescado & 80 & 70 & 60 & 60 & 50 \\
\hline Soya integral & 50 & 50 & 50 & 50 & 40 \\
\hline Torta de soya & 170 & 120 & 49 & 102 & 154 \\
\hline Polvillo de arroz & & 50 & 100 & 50 & 15 \\
\hline Afrecho de trigo & 97 & 150 & 173 & 127 & \\
\hline Melaza de caña & 40 & 40 & 40 & 40 & 25 \\
\hline Carbonato de calcio & 11 & 13.2 & 18.2 & 57 & 101 \\
\hline Aceite de soya & 10 & 10 & & 10 & 30 \\
\hline Sal común & 2.6 & 2.4 & 2.6 & 2.3 & 2.9 \\
\hline Premezcla Levante $^{1}$ & 1 & 1 & 1 & & \\
\hline Premezcla Postura $^{2}$ & & & & 1 & 1 \\
\hline Cloruro de colina $60 \%$ & 1 & 1 & 0.8 & 0.5 & 0.6 \\
\hline DL Metionina & 0.4 & & & 0.2 & 0.6 \\
\hline Bicarbonato de sodio & 1 & 1 & 1.1 & 1 & 1 \\
\hline Fosfato monodicálcico & 9 & 7.4 & 6.3 & 10 & 8.9 \\
\hline Total & 1000 & 1000 & 1000 & 1000 & 1000 \\
\hline \multicolumn{6}{|l|}{ Valores calculados ${ }^{3}$} \\
\hline $\begin{array}{l}\text { Energía metabolizable } \\
(\mathrm{kcal} / \mathrm{kg})\end{array}$ & 2850 & 2850 & 2900 & 2900 & 2900 \\
\hline Proteína cruda $(\%)$ & 18.00 & 16.00 & 15.00 & 17.00 & 15.00 \\
\hline Lisina $(\%)$ & 0.85 & 0.60 & 0.45 & 0.62 & 0.58 \\
\hline Metionina $(\%)$ & 0.30 & 0.25 & 0.20 & 0.22 & 0.25 \\
\hline Treonina $(\%)$ & 0.68 & 0.57 & 0.37 & 0.17 & 0.39 \\
\hline Ácido linoleico (\%) & 1.00 & 1.00 & 1.00 & 1.00 & 1.00 \\
\hline Calcio $(\%)$ & 0.90 & 0.80 & 0.80 & 2.00 & 3.25 \\
\hline Fósforo disponible (\%) & 0.40 & 0.35 & 0.30 & 0.32 & 0.21 \\
\hline
\end{tabular}

${ }^{1}$ Cada kg contiene: Vit. A 8000 mil UI, Vit. D 2000 mil UI, Vit. E 10000 UI, Vit. K3 2.5 g, tiamina 1.5 g, riboflavina $5 \mathrm{~g}$, piridoxina $1.5 \mathrm{~g}$, cianocobalamina $9 \mathrm{mg}$, ácido pantoténico $5 \mathrm{~g}$, ácido fólico $200 \mathrm{mg}$, niacina 25 mg, Mn 60 g, Zn 30 g, Fe 30 g, Cu 1.5 g, I 1 g, Se 100 mg, Co 100 mg

${ }^{2}$ Cada kg contiene: Vit. A 9000 mil UI, Vit. D 2000 mil UI, Vit. E 8000 UI, Vit. K3 2 g, riboflavina 10 g, cianocobalamina $10 \mathrm{mg}$, ácido pantoténico $5 \mathrm{~g}$, ácido fólico $500 \mathrm{mg}$, niacina $15 \mathrm{mg}, \mathrm{Mn} 55 \mathrm{~g}, \mathrm{Zn} 45 \mathrm{~g}$, Fe 35 g, Cu 5 g, I 1 g, Se 100 mg

${ }^{3}$ Los valores se calcularon a partir de NRC (1994)

naron dos gallinas por corral para la incubación natural. A cada gallina se le asignaron 12 huevos. La incubación duró 21 días aproximadamente, evaluándose el porcentaje de incubabilidad, de HNE, de HPEA respecto del total de HNE, y de mortalidad embrionaria.

\section{Análisis Estadístico}

Los parámetros de crecimiento de los pollos procedentes de gallina criolla chotanade huevos verdes (GCCHHV) fueron estimados mediante estadística descriptiva. Los indicadores de producción y peso de huevo 
Cuadro 2. Comportamiento productivo de pollos nacidos de huevos de cáscara verde de gallina criolla procedente de la provincia de Chota, Cajamarca, según sexo y edad

\begin{tabular}{lccc}
\hline Parámetros & $\begin{array}{c}\text { Sin sexar } \\
\left(1-10 \mathrm{sem}^{1}\right)\end{array}$ & $\begin{array}{c}\text { Machos } \\
\left(11-16 \mathrm{sem}^{2}\right)\end{array}$ & $\begin{array}{c}\text { Hembras } \\
\left(11-16 \mathrm{sem}^{2}\right)\end{array}$ \\
\hline $\begin{array}{l}\text { Ganancia media diaria } \\
\text { g/ave/día) }\end{array}$ & $17.34 \pm 0.55$ & $42.02 \pm 3.10$ & $26.31 \pm 2.21$ \\
Ingesta de alimento (g/ave/día) & $49.11 \pm 0.58$ & $139.83 \pm 0.67$ & $89.22 \pm 0.71$ \\
Conversión alimenticia & $2.83 \pm 0.07$ & $3.34 \pm 0.25$ & $3.41 \pm 0.27$ \\
Mortalidad (\%) $(\mathrm{n}=390)$ & 3.07 & -- & -- \\
\hline
\end{tabular}

${ }^{1}$ Promedio y desviación estándar de 10 repeticiones (39 pollos por repetición)

${ }^{2}$ Promedio y desviación estándar de 10 repeticiones (15 pollos por repetición)

fueron sometidos al análisis de varianza, y las medias fueron comparadas con la prueba de Tukey, utilizándose el software SAS 9.1.2. La comparación de los datos de incubación artificial y natural se lo realizó mediante Prueba t de Student.

\section{Resultados y Discusión}

\section{Crecimiento}

Los parámetros de crecimiento (GMD, ingesta de alimento, conversión alimenticia y mortalidad de pollos) se describen en el Cuadro 2. La GMD en aves sin sexar durante las 10 primeras semanas fue de $17.34 \mathrm{~g} / \mathrm{ave}$, mientras que los machos mostraron mejor GMD que las hembras en el periodo 11-16 semanas, coincidiendo con el mayor consumo de alimento por los machos. Sin embargo, al evaluar la conversión alimenticia se encontró que las hembras consumieron $70 \mathrm{~g}$ adicionales en comparación con los machos por cada kilogramo de peso ganado. Los pesos promedio fueron de $1.256 \pm 39 \mathrm{~g}$ a las 10 semanas en el lote mixto, y de $3.071 \pm 128 \mathrm{~g}$ en machos y $2.312 \pm 98 \mathrm{~g}$ en hembras a las 16 semanas. Casos de mortalidad se presentaron únicamente durante las primeras 10 semanas de vida. La curva de crecimiento semanal durante las primeras 16 semanas se muestran en la Figura 1.

Los pesos de las pollas a las 16 semanas se sitúan entre el rango 1.20 y $2.37 \mathrm{~kg}$, que se reporta para gallinas criollas ecuatorianas en edad reproductiva (Villacis et al., 2016), aunque cercano al límite superior del rango de pesos de los biotipos ecuatorianos. Así mismo, los pesos promedio de pollos criollos machos y hembras de GCHHV fueron superiores a aquellos de aves criollas de las comunidades de Oaxaca, México (LuisChincoya et al., 2016), quienes encontraron gallinas de primer periodo de postura con $1.93 \mathrm{~kg}$ y gallos jóvenes de $2.72 \mathrm{~kg}$, y con mayor coeficiente de variación, lo que significa una variación de más de $500 \mathrm{~g}$ respecto del peso vivo promedio.

Los gallos de razas autóctonas de Galicia, España (Franco et al., 2012) pesaron $3.27 \mathrm{~kg}$ a las 24 semanas de edad, con pesos máximos de $3.504 \pm 193 \mathrm{~g}$ cuando fueron alimentados solo con forraje y de $3.398 \pm$ $169 \mathrm{~g}$ con alimentación a base de maíz amarillo. El pollo de GCCHHV pesó $3.071 \mathrm{~g}$ a las 16 semanas con un desvío estándar similar al de las aves españolas. Aves de razas locales italianas de genotipo Berlanda sobrepasaron los 2 y $2.5 \mathrm{~kg}$ de peso a los 121 días en hem- 

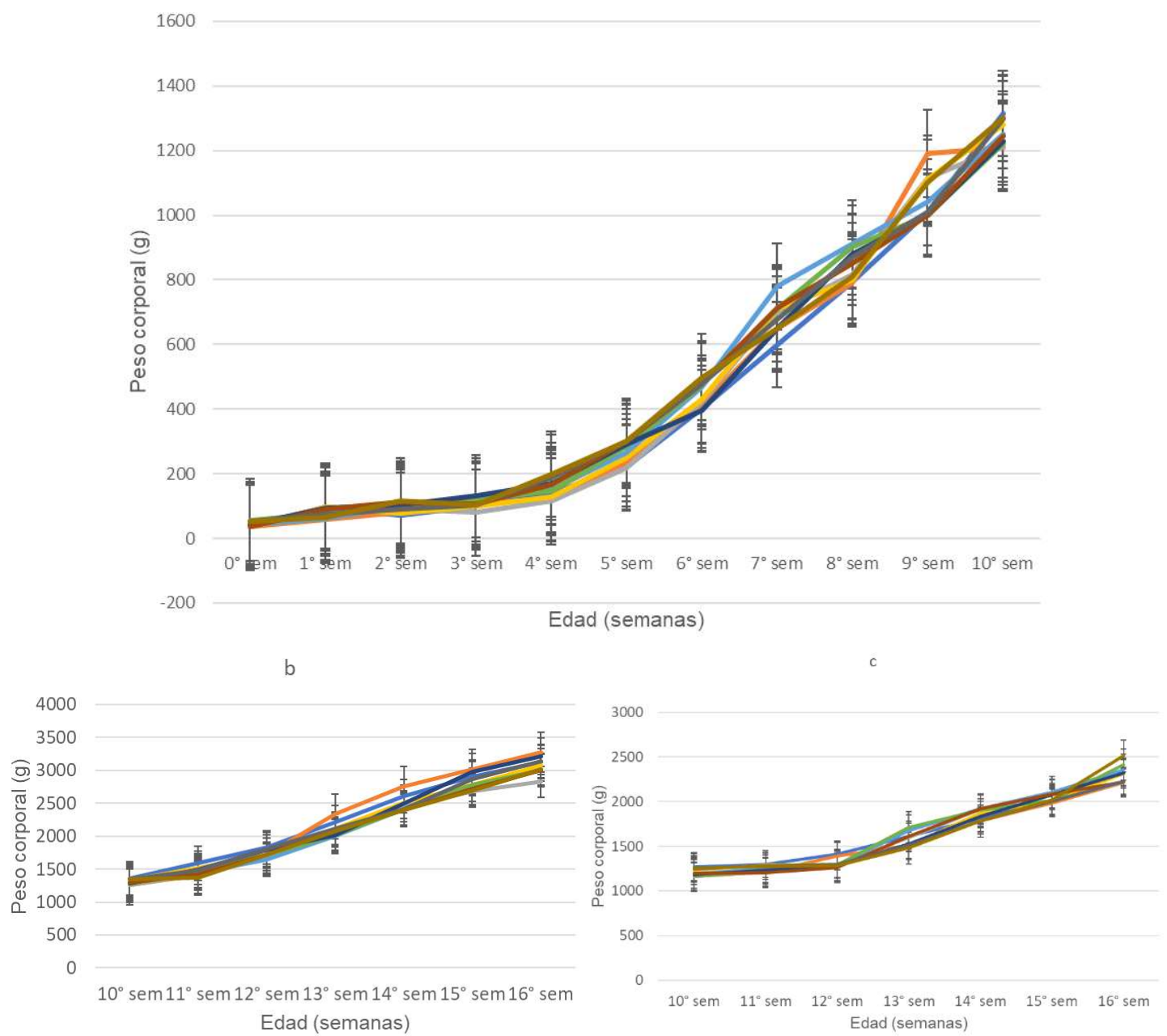

Figura 1. Curva de crecimiento de pollos nacidos de huevos de cáscara verde de gallina criolla procedente de la provincia de Chota (Perú) hasta las 16 semanas de edad de: (a) pollos sin sexar ( $\mathrm{n}=10$ repeticiones de 39 pollos por repetición); (b) pollos machos $(\mathrm{n}=10$ repeticiones de 15 pollos por repetición) y (c) pollos hembras ( $\mathrm{n}=10$ repeticiones de 15 pollos por repetición). Las barras indican el error estándar

bras y machos, respectivamente; en tanto que las aves de la variedad Padovana pesaron cerca de $1.5 \mathrm{~kg}$ de peso a los 121 días, sin diferencias entre sexos (Rizzi et al., 2013). Estas coincidencias y cercanías en cuanto a peso entre las aves criollas peruanas GCCHHV con las ecuatorianas, mexicanas, españolas e italianas permite suponer cierta cercanía genética.

Al comparar genotipos de pollos criollos de Asia se encuentran mayores diferen- cias en el tamaño de las aves. Así, en razas autóctonas saudíes se reportan pesos corporales a la madurez sexual entre $992.8 \mathrm{y}$ 1129.2 g (Fathi et al., 2017). Pollos autóctonos de la India de la variedad Aseel presentan pesos de $1.872 \mathrm{~g}$ en machos $\mathrm{y}$ $1.303 \mathrm{~g}$ en hembras a las 24 semanas, mientras que en la variedad Kadaknath, genotipo más pequeño, presentan pesos de $1.231 .4 \mathrm{~g}$ en machos y $936.9 \mathrm{~g}$ en hembras a las 24 semanas (Haunshi et al., 2011). Por otro lado, pollos de la región montañosa de Erlang, Chi- 
Cuadro 3. Producción de huevos $(\%)^{1}$ de cáscara verde de gallina criolla procedente de la provincia de Chota, Cajamarca, durante dos fases de postura y según biotipo (inicio de postura: 17 semanas)

\begin{tabular}{|c|c|c|c|c|c|c|c|}
\hline \multirow{2}{*}{$\begin{array}{l}\text { Edad } \\
(\text { sem })\end{array}$} & \multicolumn{6}{|c|}{ Biotipo } & \multirow{2}{*}{$\begin{array}{c}P- \\
\text { value }\end{array}$} \\
\hline & Negro & Castaño & Colorado & Blanco & $\begin{array}{c}\text { Cuello } \\
\text { desnudo }\end{array}$ & Riña & \\
\hline \multicolumn{8}{|c|}{ Primera fase de postura ${ }^{2}$} \\
\hline 17 & $5.7 \pm 2.3^{\mathrm{b}}$ & $4.3 \pm 1.7^{b}$ & $2.9 \pm 2.3^{\mathrm{a}}$ & -- & -- & -- & 0.003 \\
\hline 18 & $48.6 \pm 4.7^{b}$ & $46.0 \pm 5.1^{\mathrm{b}}$ & $48.6 \pm 7.0^{\mathrm{b}}$ & $12.9 \pm 3.7^{\mathrm{a}}$ & $11.4 \pm 6.2^{\mathrm{a}}$ & -- & 0.0003 \\
\hline 19 & $70.0 \pm 8.3^{\mathrm{c}}$ & $72.9 \pm 6.8^{\mathrm{c}}$ & $70.0 \pm 6.8^{\mathrm{c}}$ & $48.6 \pm 11.9^{b}$ & $50.0 \pm 6.0^{\mathrm{b}}$ & $11.4 \pm 5.2^{\mathrm{a}}$ & 0.0004 \\
\hline 20 & $70.0 \pm 8.3^{\mathrm{b}}$ & $72.9 \pm 6.8^{b}$ & $70.0 \pm 8.9^{\mathrm{b}}$ & $70.0 \pm 6.0^{\mathrm{b}}$ & $70.0 \pm 10.6^{\mathrm{b}}$ & $41.4 \pm 5.9^{\mathrm{a}}$ & 0.002 \\
\hline 21 & $70.0 \pm 5.9^{b}$ & $72.9 \pm 6.0^{\mathrm{b}}$ & $70.0 \pm 8.9^{b}$ & $70.0 \pm 6.8^{b}$ & $70.0 \pm 11.1^{\mathrm{b}}$ & $51.4 \pm 14.0^{\mathrm{a}}$ & 0.003 \\
\hline 22 & $65.7 \pm 7.0^{\mathrm{b}}$ & $62.9 \pm 10.4^{\mathrm{b}}$ & $67.1 \pm 12.0^{\mathrm{b}}$ & $70.0 \pm 3.7^{b}$ & $68.6 \pm 9.3^{\mathrm{b}}$ & $51.4 \pm 5.2^{\mathrm{a}}$ & 0.003 \\
\hline 23 & $20.0 \pm 2.3^{\mathrm{a}}$ & $14.3 \pm 2.3^{\mathrm{a}}$ & $19.8 \pm 5.1^{\mathrm{a}}$ & $28.6 \pm 9.3^{b}$ & $40.0 \pm 7.0^{\mathrm{c}}$ & $34.3 \pm 2.3^{\mathrm{b}}$ & 0.009 \\
\hline \multicolumn{8}{|c|}{ Segunda fase de postura ${ }^{3}$} \\
\hline 27 & $41.4 \pm 8.2^{c}$ & $57.1 \pm 4.7^{\mathrm{c}}$ & $50.0 \pm 3.7^{\mathrm{c}}$ & $41.4 \pm 8.2^{c}$ & $24.3 \pm 8.2^{b}$ & $15.7 \pm 3.7^{\mathrm{a}}$ & 0.001 \\
\hline 28 & $70.0 \pm 10.6^{\mathrm{b}}$ & $72.9 \pm 3.7^{b}$ & $70.0 \pm 10.0^{\mathrm{b}}$ & $70.0 \pm 8.9^{b}$ & $70.0 \pm 10.6^{\mathrm{b}}$ & $42.9 \pm 7.0^{\mathrm{a}}$ & 0.002 \\
\hline 29 & $70.0 \pm 6.0^{\mathrm{b}}$ & $72.9 \pm 6.0^{\mathrm{b}}$ & $71.4 \pm 11.2^{\mathrm{b}}$ & $70.7 \pm 12.4^{b}$ & $70.7 \pm 10.0^{\mathrm{b}}$ & $45.7 \pm 9.3^{\mathrm{a}}$ & 0.002 \\
\hline 30 & $70.0 \pm 8.3^{\mathrm{b}}$ & $72.9 \pm 8.3^{b}$ & $70.0 \pm 3.7^{b}$ & $70.0 \pm 8.3^{\mathrm{b}}$ & $70.7 \pm 11.0^{\mathrm{b}}$ & $35.7 \pm 3.7^{\mathrm{a}}$ & 0.001 \\
\hline 31 & $70.0 \pm 8.9^{\mathrm{b}}$ & $73.6 \pm 14.36^{\mathrm{b}}$ & $70.7 \pm 9.4^{\mathrm{b}}$ & $70.0 \pm 8.3^{\mathrm{b}}$ & $70.0 \pm 3.7^{b}$ & $50.0 \pm 5.9^{\mathrm{a}}$ & 0.003 \\
\hline 32 & $63.6 \pm 3.6^{\mathrm{b}}$ & $55.7 \pm 10.6^{\mathrm{a}}$ & $67.1 \pm 13.3^{\mathrm{b}}$ & $70.0 \pm 10.6^{\mathrm{b}}$ & $70.0 \pm 8.9^{b}$ & $50.0 \pm 5.4^{\mathrm{a}}$ & 0.003 \\
\hline 33 & $12.9 \pm 3.7^{\mathrm{a}}$ & $12.9 \pm 1.6^{\mathrm{a}}$ & $11.4 \pm 2.3^{\mathrm{a}}$ & $25.7 \pm 2.3^{\mathrm{b}}$ & $22.9 \pm 8.4^{\mathrm{b}}$ & $24.3 \pm 3.6^{\mathrm{b}}$ & 0.0004 \\
\hline
\end{tabular}

${ }^{1}$ Promedios \pm desviación estándar de 4 repeticiones por biotipo ( 5 gallinas por repetición)

${ }^{2}$ La primera fase de postura se interrumpe a la semana 24 de edad con producciones de huevo en todos los biotipos $<10 \%$ (periodo de cloquera)

${ }^{3}$ La segunda fase de postura se interrumpe a la semana 34 de edad con producciones de huevo en todos los biotipos $<10 \%$ (periodo de cloquera con mayor persistencia)

na logran pesos promedio de 2.2 y $1.8 \mathrm{~kg}$, en machos y hembras, respectivamente, a las 10 semanas de edad (Li et al., 2013), los cuales superan al peso que alcanzan los pollos GCCHHV sin sexar (1256 g a las 10 semanas).

\section{Comportamiento Reproductivo}

El Cuadro 3 muestra que la producción de huevos durante las 14 semanas de postura. La postura estuvo afectada por el biotipo $(\mathrm{p}<0.05)$. La tasa de puesta de huevos varió acorde con la edad de inicio de la postura. Los biotipos Negro, Castaño y Colorado alcanzaron el pico de producción en la tercera semana, los biotipos Blanco y Cuello desnudo en la cuarta semana, y el biotipo Riña en la quinta semana. La postura estuvo interrumpida por un periodo de cloquera, que si bien no cesó por completo la postura, en todos los biotipos fue inferior al $10 \%$ de la producción que se venía presentando. Este periodo no sobrepasó los 21 días, lo cual coincide con Jiang et al. (2010) en gallinas nativas de la India, raza Quingyuan, que presentan cloquera entre 10 y 21 días.

Se encontraron diferencias significativas en el peso del huevo de gallina GCCHHV de acuerdo con el biotipo ( $<<0.05$; Cuadro $4)$. En ambas fases de postura se encontra- 
Cuadro 4. Peso del huevo $(\mathrm{g})^{1}$ de cáscara verde de gallina criolla procedente de la provincia de Chota, Cajamarca, durante dos fases de postura y según biotipo (inicio de postura: 17 semanas)

\begin{tabular}{|c|c|c|c|c|c|c|c|}
\hline \multirow{2}{*}{$\begin{array}{r}\text { Edad } \\
\text { (sem) }\end{array}$} & \multicolumn{6}{|c|}{ Biotipo } & \multirow{2}{*}{$\begin{array}{c}P- \\
\text { value }\end{array}$} \\
\hline & Negro & Castaño & Colorado & Blanco & $\begin{array}{c}\text { Cuello } \\
\text { desnudo }\end{array}$ & Riña & \\
\hline \multicolumn{8}{|c|}{ Primera fase de postura $^{2}$} \\
\hline 17 & $46.2 \pm 1.5^{b}$ & $47.3 \pm 0.3^{c}$ & $45.5 \pm 0.5^{\mathrm{a}}$ & -- & -- & -- & 0.003 \\
\hline 18 & $47.3 \pm 0.5^{\mathrm{a}}$ & $49.2 \pm 0.8^{b}$ & $47.1 \pm 0.3^{\mathrm{a}}$ & $47.5 \pm 0.4^{\mathrm{a}}$ & $49.1 \pm 0.2^{b}$ & -- & 0.006 \\
\hline 19 & $49.6 \pm 0.5^{b}$ & $51.1 \pm 0.5^{\mathrm{c}}$ & $49.9 \pm 0.8^{b}$ & $49.9 \pm 0.5^{b}$ & $51.6 \pm 0.4^{\mathrm{c}}$ & $44.6 \pm 0.2^{\mathrm{a}}$ & 0.0004 \\
\hline 20 & $51.4 \pm 0.1^{b}$ & $53.3 \pm 0.8^{\mathrm{c}}$ & $51.4 \pm 0.4^{b}$ & $51.1 \pm 0.4^{b}$ & $53.4 \pm 0.4^{\mathrm{c}}$ & $46.4 \pm 0.3^{\mathrm{a}}$ & 0.0004 \\
\hline 21 & $53.4 \pm 0.3^{b}$ & $55.4 \pm 0.3^{\mathrm{c}}$ & $53.1 \pm 0.9^{b}$ & $53.3 \pm 0.4^{b}$ & $55.1 \pm 0.1^{\mathrm{c}}$ & $48.1 \pm 0.6^{\mathrm{a}}$ & 0.0004 \\
\hline 22 & $55.1 \pm 0.3^{b}$ & $57.3 \pm 0.6^{\mathrm{c}}$ & $55.2 \pm 0.6^{\mathrm{b}}$ & $55.2 \pm 0.7^{b}$ & $57.2 \pm 0.1^{\mathrm{c}}$ & $50.8 \pm 0.5^{\mathrm{a}}$ & 0.0004 \\
\hline 23 & $56.4 \pm 0.3^{b}$ & $58.4 \pm 0.4^{\mathrm{c}}$ & $56.7 \pm 0.2^{\mathrm{b}}$ & $56.6 \pm 0.4^{b}$ & $58.5 \pm 0.3^{\mathrm{c}}$ & $52.9 \pm 0.8^{\mathrm{a}}$ & 0.0004 \\
\hline \multicolumn{8}{|c|}{ Segunda fase de postura ${ }^{3}$} \\
\hline 27 & $57.2 \pm 0.7^{b}$ & $59.5 \pm 0.2^{\mathrm{c}}$ & $57.4 \pm 0.1^{b}$ & $57.4 \pm 0.6^{b}$ & $59.3 \pm 0.5^{c}$ & $53.9 \pm 0.2^{\mathrm{a}}$ & 0.0003 \\
\hline 28 & $58.3 \pm 0.1^{\mathrm{b}}$ & $60.3 \pm 0.4^{\mathrm{c}}$ & $58.4 \pm 0.3^{b}$ & $58.6 \pm 0.6^{b}$ & $60.6 \pm 0.2^{\mathrm{c}}$ & $54.8 \pm 0.4^{\mathrm{a}}$ & 0.0004 \\
\hline 29 & $59.7 \pm 0.2^{\mathrm{b}}$ & $61.5 \pm 0.3^{\mathrm{c}}$ & $59.5 \pm 0.2^{\mathrm{b}}$ & $59.2 \pm 0.5^{b}$ & $61.6 \pm 0.4^{\mathrm{c}}$ & $55.3 \pm 0.1^{\mathrm{a}}$ & 0.0004 \\
\hline 30 & $60.7 \pm 0.3^{b}$ & $62.4 \pm 0.4^{\mathrm{c}}$ & $60.4 \pm 0.2^{\mathrm{b}}$ & $60.5 \pm 0.4^{b}$ & $62.7 \pm 0.3^{\mathrm{c}}$ & $56.3 \pm 0.5^{\mathrm{a}}$ & 0.0004 \\
\hline 31 & $61.2 \pm 0.8^{\mathrm{b}}$ & $63.4 \pm 0.2^{\mathrm{c}}$ & $61.1 \pm 0.7^{b}$ & $61.3 \pm 0.1^{b}$ & $63.3 \pm 0.5^{\mathrm{c}}$ & $57.1 \pm 0.4^{\mathrm{a}}$ & 0.0004 \\
\hline 32 & $61.5 \pm 0.5^{\mathrm{b}}$ & $63.5 \pm 0.3^{\mathrm{c}}$ & $61.6 \pm 0.4^{\mathrm{b}}$ & $61.5 \pm 0.6^{b}$ & $63.2 \pm 0.3^{\mathrm{c}}$ & $57.8 \pm 0.1^{\mathrm{a}}$ & 0.0039 \\
\hline 33 & $62.3 \pm 0.5^{\mathrm{b}}$ & $64.4 \pm 0.3^{\mathrm{c}}$ & $62.5 \pm 0.3^{\mathrm{b}}$ & $62.4 \pm 0.6^{b}$ & $64.3 \pm 0.4^{\mathrm{c}}$ & $58.3 \pm 0.3^{\mathrm{a}}$ & 0.0004 \\
\hline
\end{tabular}

${ }^{1}$ Promedios \pm desviación estándar de 4 repeticiones por biotipo ( 5 gallinas por repetición)

${ }^{2}$ La primera fase de postura se interrumpe a la semana 24 de edad con producciones de huevo en todos los biotipos $<10 \%$ (periodo de cloquera)

${ }^{3}$ La segunda fase de postura se interrumpe a la semana 34 de edad con producciones de huevo en todos los biotipos $<10 \%$ (periodo de cloquera con mayor persistencia)

ron huevos con mayor peso a favor del biotipo Cuello Desnudo y el menor peso de huevo en el biotipo Riña. El peso del huevo de estos genotipos durante las tres primeras semanas de postura se asemeja al de la gallina criolla ecuatoriana con $50.5 \mathrm{~g}$ (Andrade-Yucailla et al., 2015), alimentadas con grano de maíz. El peso promedio del huevo de la gallina GCHHV fue de 51.8 y $60.2 \mathrm{~g}$ en la primera $\mathrm{y}$ segunda fase de postura, similares a los reportados por Rizzi y Marangon (2012) en gallinas nativas italianas Ermellinata de Rovigo y Robusta Maculata, con pesos de 54.4 y 56.5 $\mathrm{g}$, respectivamente, bajo crianza al pastoreo con suplementación de alimento balanceado. Por otro lado, las gallinas asiáticas Aseel producen huevos con pesos menores (42.7 y $49.3 \mathrm{~g}$ en las semanas 28 y 40 de edad) (Haunshi et al., 2011) y la gallina Saudí produce huevos entre 38 y $40.2 \mathrm{~g}$ (Fathi et al., 2017). Se puede inferir que la gallina criolla a menor peso corporal tiende a producir huevos menos pesados; por tanto, la gallina GCCHHV por ser una gallina de mayor peso $(>2.5 \mathrm{~kg}$ en la segunda fase de postura) produce huevos con más de $60 \mathrm{~g} \mathrm{y}$, considerando su masa corporal, la hace, también, muy apreciada para el aprovechamiento de su carne. 
Cuadro 5. Incubabilidad del huevo de cáscara verde de gallina criolla procedente de la provincia de Chota, Cajamarca, en incubación artificial e incubación natural según biotipo

\begin{tabular}{|c|c|c|c|c|c|c|c|c|c|}
\hline \multirow[b]{2}{*}{ Indicador } & \multirow{2}{*}{ 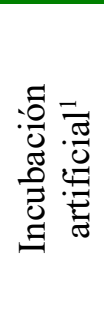 } & \multicolumn{6}{|c|}{$\begin{array}{c}\text { Incubación natural }^{2}(\mathrm{n}=6 \text { repeticiones de } 96 \\
\text { huevos cada una) } \\
\text { Biotipo }\end{array}$} & \multirow{2}{*}{$\begin{array}{c}\text { Media } \pm \\
\text { D.E. }\end{array}$} & \multirow{2}{*}{$\begin{array}{c}\mathrm{P}- \\
\text { value }\end{array}$} \\
\hline & & $\begin{array}{l}\stackrel{0}{b} \\
\ddot{C}\end{array}$ & Ů. & $\begin{array}{l}\frac{8}{\pi} \\
0 \\
0 \\
0\end{array}$ & 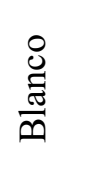 & 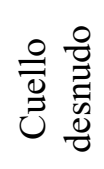 & 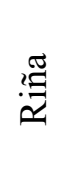 & & \\
\hline Peso del huevo, $\mathrm{g}$ & 58.8 & 60.1 & 62.1 & 60.1 & 60.1 & 62.1 & 58.7 & $60.6 \pm 1.4$ & 0.021 \\
\hline $\mathrm{HNE}^{3}, \%$ & 22.0 & 11.7 & 13.8 & 13.8 & 15.6 & 20.8 & 21.9 & $16.3 \pm 4.1$ & 0.014 \\
\hline $\mathrm{HPEA}^{4}, \%$ de HNE & 54.6 & 54.6 & 53.9 & 23.1 & 70.0 & 80.0 & 50.0 & $55.2 \pm 19.5$ & 0.817 \\
\hline $\mathrm{MEFI}^{5}, \%$ de 500 & 10 & 5.3 & 6.4 & 10.6 & 4.7 & 4.2 & 10.9 & $7.0 \pm 3.0$ & 0.052 \\
\hline Incubabilidad $^{6}, \%$ & 78 & 88.3 & 86.2 & 86.2 & 84.4 & 79.2 & 78.1 & $83.7 \pm 4.1$ & 0.014 \\
\hline
\end{tabular}

${ }^{1}$ A partir de 500 huevos de cáscara verde recolectados en la provincia de Chota

${ }^{2}$ A partir de los huevos de cáscara verde producidos por las gallinas encasetadas en el valle de Cajamarca (cada gallina incubó 12 huevos)

${ }^{3}$ HNE: Huevos no eclosionados, \% del total de huevos incubados

${ }^{4}$ HPEA: Huevos putrefactos en estado acuoso, \% del total de huevos no eclosionados

${ }^{5} \mathrm{MEFI}$ : Mortalidad embrionaria al final de la incubación, \% del total de huevos incubados

${ }^{6}$ Incubabilidad: \% de pollos nacidos vivos en relación al total de huevos incubados

Las gallinas GCCHHV, encasetadas y alimentadas con dietas de postura, produjeron huevos con mayor porcentaje de incubabilidad ( 83.7 vs. $78.0 \%$ ) que el tratamiento con huevos colectados in situ y con incubación artificial (Cuadro 5), posiblemente debido a las diferencias en el peso del huevo incubable $(\mathrm{p}<0.05)$ y a otros factores de manejo en la producción y manipulación del huevo. En forma similar, hubo un menor porcentaje de HNE que en los huevos provenientes de incubación natural $(\mathrm{p}<0.05)$; no obstante, no hubo diferencias entre los dos tratamientos con respecto al porcentaje de HPF para los grupos de incubación artificial (54.5\%) e incubación natural (55.2\%) (Cuadro 5). La mortalidad embrionaria mostró una tendencia a reducirse con la incubación natural (de 10.0 a $7.0 \%$ ), lo que puede sugerir que los huevos de estas gallinas requieren de mayor tiempo de incubación.
Los resultados de incubabilidad de la gallina GCCHHV fueron similares a los resultados de Andrade (2011) en gallina criolla ecuatoriana, quien obtuvo $79.2 \%$ de incubabilidad en huevos incubados artificialmente, obtenidos de gallinas alimentadas con dietas de postura y alojadas en corrales, fuera del hábitat natural, como se practicó con la gallina GCCHHV del presente estudio. Por otro lado, Juarez-Caratachea y Ortiz (2001) obtuvieron valores inferiores de incubabilidad (60.7\%) en huevos colectados de comunidades de Michoacán, México.

\section{Conclusiones}

- El crecimiento y tamaño de los biotipos negro, colorado, castaño, blanco y cuello desnudo procedentes de gallinas crio- 
llas procedentes de la provincia de Chota, Cajamarca (GCCHHV), fue similar, con excepción del biotipo Riña que fue menor.

- La producción de huevos de las gallinas GCCHHV se inicia entre las 17 y 19 semanas de edad, con periodos de postura de siete semanas interrumpidos por una etapa de cloquera que puede durar hasta tres semanas.

- La incubabilidad de los huevos de la gallina GCCHHV se ve influenciada por las condiciones y tipos de incubación.

\section{Literatura Citada}

1. Andrade C. 2011. Determinación de parámetros reproductivos y productivos de gallinas criollas para huevo verde, desde la recolección de huevo hasta la etapa inicial. Tesis de Ingeniero Zootecnista. Ecuador: Escuela Superior Politécnica de Chimborazo. 104 p.

2. Andrade-Yucailla V, Vargas-Burgos $J C$, Lima-Orozco $R$, Moyano $J$, Navarrete H, López J, Sánchez J. 2015. Características físicas del huevo de gallinas criolla y campera (Gallus domesticus) en la región amazónica del Ecuador. AICA 6: 49-54

3. Burley H, Anderson K, Patterson P, Tillman P. 2016. Formulation challenges of organic poultry diets with readily available ingredients and limited synthetic methionine. J Appl Poultry Res 25: 443454. doi: 10.3382/japr/pfw012

4. Dunn-Horrocks S, Pichardo-Fuchs M, Lee J, Ruiz-Feria C, Creger C, Hyatt D, Farnell M 2011. Effect ofomega-3 enriched layer rations on egg quality. Int J Poultry Sci 10: 8-11. doi: 10.3923/ ijps.2011.8.11

5. Farrell D. 2008. The role of poultry in human nutrition. Food and Agriculture Organization of the United Nations FAO. [Internet]. Available in: http:// www.fao.org/docrep/013/a1709e/ al709e00.pdf
6. Fathi MM, Al-Homidan I, Motawei MI, Abou-Emera OK, El-Zarei MF. 2017. Evaluation of genetic diversity of Saudi native chicken populations using microsatellite markers. J Poult Sci 96: 530-536. doi: 10.3382/ps/pew357

7. Franco D, Rois D, Vázquez JA, Purriños L, González R, Lorenzo JM. 2012. Breed effect between Mos rooster (Galician indigenous breed) and Sasso T-44 line and finishing feed effect of commercial fodder or corn. J Poult Sci 91: 487-498. doi: 10.3382/ ps.2011-01546

8. Geng AL, Zhang Y, Zhang J, Wang HH, Chu Q, Liu HG. 2018. Effects of lighting pattern and photoperiod on egg production and egg quality of a native chicken under free-range condition. J Poult Sci 97: 2378-2384. doi: 10.3382/ps/ pey104.

9. Gongruttananun N. 2014. Influence of red light on reproductive performance, eggshell ultrastructure, and eye morphology in Thai-native hens. Poultry Sci 90: 2855-2863.doi: 10.3382/ps.201101652

10. Haunshi S, Niranjan M, Shanmugam M, Padhi MK, Reddy MR, Sunitha R, Rajkumar U, et al. 2011. Characterization of two Indian native chicken breeds for production, egg and semen quality, and welfare traits. J Poult Sci 90: 314-320. doi: 10.3382/ps.2010-01013

11. Jiang RS, Chen XY, Geng ZY. 2010. Broodiness, egg production, and correlations between broody traits in an indigenous chicken breed. J Poult Sci 89: 1094-1096. doi: 10.3382/ps.2009-00621

12. Juárez-Caratachea A, Ortiz M. 2001. Estudio de la incubabilidad y crianza de las aves traspatio. Vet Méx 32: 27-32.

13. Li J, Zhao XL, Yuan YC, Gilbert ER, Wang Y, Liu YP, Zhang Y, Zhu $Q$. 2013. Dietary lysine affects chickens from local Chinese pure lines and their reciprocal crosses. J Poult Sci 92: 16831689. doi: $10.3382 /$ ps.2012-02865. 
14. Luis-Chincoya H, Jerez-Salas MP, Herrera-Haro JG, Mendoza-Nazar P. 2016. Caracterización fenotípica y sistema de producción de las gallinas criollas en comunidades de Oaxaca. Rev Mex Agroecosistemas 3: 87-98.

15. Mugnai C, Sossidou E, Dal Bosco A, Ruggeri S, Mattioli S, Castellini C. 2014. The effects of husbandry system on the grass intake and nutritive characteristics of laying hens. J Sci Food Agr 94: 459-467. doi: 10.1002/jsfa.6269

16. [NRC] National Research Council. 1994. Nutrient requirements of poultry. $9^{\text {th }}$ rev. ed. Washington DC: Acad. Press. $176 \mathrm{p}$.

17. Osman, S, Yonezawa T, Nishibori M. 2016. Origin and genetic diversity of Egyptian native chickens based on complete sequence of mitochondrial DNA Dloop region. Poultry Sci 95: 1248-1256. doi: $10.3382 / \mathrm{ps} /$ pew029

18. Quintana JA. 2013. Avitecnia. $4^{\text {a }}$ ed. México DF: Ed Trillas. 406 p.

19. Rizzi C, Marangon A. 2012. Quality of organic eggs of hybrid and Italian breed hens. J Poult Sci 91: 2330-2340. doi: 10.3382/ps.2011-01966
20. Samiullah S, Roberts J, Chousalkar K. 2016. Oviposition time, ûock age, and egg position in clutch in relation to brown eggshell color in laying hens. Poultry Sci 95: 2052-2057. doi: 10.3382/ps/pew197

21. Sánchez M. 2012. Caracterización local de gallinas criollas (Gallus domesticus) encontradas en los traspa-tios de las familias de Tomás Garrido, Tacotalpa, Tabasco. 31 p. [Internet]. Disponible en: https://es.scribd.com/document/.../010124a-Informe-Gallinas-RosarioSanchez-1.pdf

22. Villacís G, Escudero G, Cueva F, Luzuriaga, A. 2014. Características morfométricas de las gallinas criollas de comunidades rurales del sur del Ecuador. Rev Inv Vet Perú 27: 218-224. 10.15381/rivep.v27i2.11639

23. Wang X, Zhao C, Li J, Xu G, Lian L, $W u$ C, Deng X. 2009. Comparison of the total amount of eggshell pigments in Dongxiang brown-shelled eggs and Dongxiang blue-shelled eggs. Poultry Sci 88: 1735-1739. doi: 10.3382/ps.2008-00434

24. Wilson P. 2017. Recent advances in avian egg science: a review. J Poult Sci 96: 3747-3754. doi: 10.3382/ps/pex187 